\title{
MARINA ABRAMOVIC, créatrice en Art corporel. Eléments d'une biographie
}

Entretien réalisé par Sophie CASSAGNES-BROUQUET à l'occasion de la rétrospective organisée à la Galerie Serge Le Borgne à Paris le 7 février 2009

\section{(2) OpenEdition}

Édition électronique

URL : http://journals.openedition.org/clio/9469

DOI : 10.4000/clio.9469

ISSN : 1777-5299

Éditeur

Belin

Édition imprimée

Date de publication : 15 décembre 2009

Pagination : 195-210

ISSN : 1252-7017

\section{Référence électronique}

"MARINA ABRAMOVIc, créatrice en Art corporel. Eléments d'une biographie», Clio. Histoire, femmes et

sociétés [En ligne], 30 | 2009, mis en ligne le 15 décembre 2012, consulté le 15 septembre 2020. URL : http://journals.openedition.org/clio/9469 


\section{Témoignage}

\section{MARINA ABRAMOVIC, créatrice en Art corporel}

\section{Eléments d'une biographie}

Marina Abramović est née à Belgrade en 1946. Elle étudie à l'Académie des Beaux-Arts de Belgrade de 1965 à 1970 et choisit rapidement l'Art corporel. Elle s'est lacérée, flagellée, a congelé son corps sur des blocs de glace, pris des produits psycho-actifs et de contrôle musculaire, elle s'est mise en danger. Cependant, le but de cette artiste n'est pas le sensationnel. Ses œuvres sont autant d'expériences et de tentatives de redéfinition des limites : contrôle de son propre corps, rapports à un interprète, à l'art et aux codes qui régissent la société. Ses premières œuvres témoignent déjà d'une rébellion contre son éducation stricte et la culture répressive de la Yougoslavie communiste. Ainsi, en 1974, elle met le feu à une étoile soviétique sur laquelle elle s'est couchée (Rythm 5). Plus tard, lors de la performance Thomas Lips, elle se taille la même étoile sur le ventre avec une lame de rasoir.

Ces actions se veulent des rituels de purification, conçus pour sa propre libération. Lors d'un happening à Naples, elle livre littéralement son corps au public, auquel elle annonce : "Faites de moi ce que vous voulez», mettant à sa disposition des outils de torture (couteaux, haches, seringues, fouets), mais aussi des fleurs : elle est maltraitée, ses vêtements arrachés, on braque sur elle un pistolet chargé. En 1975 débute sa collaboration avec Ulay, un artiste allemand, le couple mythique s'installe à Amsterdam. Ensemble ils pratiquent des Relationworks, dans lesquels ils interrogent les rapports homme-femme, se fixant l'un l'autre pendant des heures, s'envoyant des gifles. Pendant deux décennies de vie commune, ils explorent les 
rapports de pouvoir et de dépendance dans une relation triangulaire avec le public. En 1989, Marina tente à l'aide d'installations composées de bois, de cristaux et de pierres de passer de l'état de conscience individuelle à celui de conscience collective. Au début des années 1990, elle confond son corps à la terre dans une série de performances : cinq serpents lui rampent sur le corps, enserrent son visage. En 1993, elle monte à Francfort The Biography, une performance dans laquelle elle visualise les étapes de sa carrière d'artiste. À l'occasion de la Biennale de Venise, Marina Abramovic crée l'évènement avec Balkan Baroque. Bouleversée par le déchirement de la guerre en Yougoslavie, elle reste assise 4 jours pendant 6 heures sur un énorme tas d'os sanglants, chante des lamentations et râpe les os pour en enlever des lambeaux de viande. Marina Abramovic a récemment acquis à Hudson près de New York un théâtre pour y installer sa Fondation pour la préservation de l'Art de la Performance. Elle souhaite en faire un lieu de rencontre et de résidence pour les artistes, les performeurs, les historiens et critiques d'art intéressés par l'art de la performance. En parallèle à sa carrière artistique, Marina Abramovic a toujours enseigné : Académie des Beaux-Arts de Novi Sad de 1973 à 1975, en 1990-1991 à Berlin et à Paris, de 1992 à 1995 à l'Ecole supérieure des Beaux-Arts d'Hambourg.

Entretien réalisé par Sophie CASSAGNES-BrouQuet à l'occasion de la rétrospective organisée à la Galerie Serge Le Borgne à Paris le 7 février 2009

Clio tient à exprimer tous ces remerciements à Serge Le Borgne et Marianne Derrien.

Clio : Pour commencer, nous souhaiterions vous demander si vous vous considérez vous-même comme une sorte d'héroïne dans le domaine de l'art, ou si, tout simplement tous les artistes, tous les performeurs ne sont pas des héros?

MA : C'est une question vraiment très complexe car, pour ma part, en tant que personne privée, je ne me considère pas du tout comme une 
héroïne, en aucune façon car cela supposerait que je me place dans une position égale à celle d'un artiste avec un grand A. Vous savez au Moyen Âge, on ne savait rien de la notion d'artiste, les artistes étaient anonymes, on ne connaît même pas leurs noms, s'ils étaient des hommes ou des femmes, où ils travaillaient; ce qui était important c'était l'œuvre d'art. Pour ma part, je considère que c'est l'art qui est important, l'art est un outil, un langage pour exprimer différentes choses et c'est ce que j'essaie de transmettre, mais personnellement je ne me considère pas comme une héroïne. C'est très différent de ce que j'essaie de transmettre. Il y a une grande différence entre la personne publique et la personne privée, ce doit être différent et c'est différent.

Clio : Le terme d'héroïne est ambigu car il a deux significations, la femme qui a eu un geste ou un comportement héroïque, mais aussi l'héroïne de fiction, de roman.

MA : Oh oui, mais je pense vraiment que cette appréciation doit être celle des critiques ou des historiens de l'art et de la position dans laquelle ils interprètent mon travail, mon art ou moi-même. Mais je pense qu'en tant qu'artiste, il est très important de conserver une certaine modestie. Au moment où vous commencez à vous considérer comme une héroïne, vous devenez une sorte d'icône, une position tout à fait erronée qui pourrait faire oublier votre création.

\section{Clio : La création est-elle un acte héroïque?}

MA : Vous savez, c'est très différent de la façon dont je considère l'héroïsme ;il nous faudrait définir une conception de l'héroïsme. Je pense que les héros sont des êtres humains capables d'aller jusqu'à sacrifier leur propre existence au bénéfice des autres. C'est ma définition de l'héroïsme. Se sacrifier soi-même, même confronté avec la possibilité de la mort, c'est ça l'hérö̈sme. C'est une curieuse histoire, mais très intéressante car je ne l'ai jamais raconté à personne, l'histoire de ma mère qui était une hérö̈ne, elle faisait partie des partisans à l'époque de Tito, mais elle ne m'en avait jamais parlé de 
toute sa vie, j'ignore pourquoi ; ce n'est qu'à sa disparition que j'ai trouvé dans ses objets personnels et dans les journaux qu'elle était une héroïne. C'est l'histoire que je voudrais vous raconter maintenant.

\section{Clio : Mais pourquoi ? L'avait-elle occulté, volontairement ?}

MA : Non, mais je crois qu'elle ne voulait pas parler de la guerre, c'était très difficile, des souvenirs trop durs, elle ne voulait pas parler de guerre ni d'héroïsme, tout comme mon père, je ne l'ai appris que par d'autres personnes à son propos.

Le cœur de l'histoire, c'est qu'elle avait alors seulement 23 ans et elle appartenait à un commando sanitaire ; elle se trouvait sur la ligne de front au milieu des explosions et des grenades. Elle s'occupait des blessés, les amenant de la ligne de front à l'hôpital, c'était sa responsabilité, son devoir avec son groupe. C'était pendant la libération de Belgrade en 1945, vers la fin de la guerre. Il y avait des combats très violents entre les Allemands et les partisans. Une partie de Belgrade sur les collines était libérée et pas le reste, et elle était dans un camion avec six autres infirmières; il y avait un violent combat de rues, les partisans se trouvaient sous le feu, en particulier dans la zone où était situé l'hôpital. À un moment le chauffeur a été tué et le camion est resté au milieu et a pris feu; elle se trouvait dedans avec quarante cinq partisans gravement blessés, elle a donc du sortir dans les rues en feu avec les six autres infirmières qui emportaient les blessés, mais elle a couru vers le camion en flammes avant qu'il n'explose pour prendre la radio pour demander à l'hôpital d'envoyer un autre camion pour sauver toutes ces personnes. Un autre camion est arrivé et les infirmières ont commencé à charger les blessés pour les conduire à l'hôpital, mais entre-temps les combats étaient tels que quatre infirmières étaient déjà mortes et qu'il ne restait plus qu'elle et une infirmière. Elle devait avoir une sorte de force surnaturelle pour réussir à tous les monter dans le camion et les conduire jusqu'aux collines et à l'hôpital. Et voilà pourquoi ma mère est une héroïne. Mais vous savez, je n'ai jamais rien su de cette histoire toute ma vie et je ne l'ai appris qu'à sa mort. Elle est morte l'année dernière. Vous voyez, c'est elle la vraie héroïne pour moi. 
Et vous voyez, c'est cette sorte de relation que je veux aussi avoir, en explorant les frontières de l'œuvre, du langage et utilisant le corps comme il ne l'a jamais été, c'est un travail que nous devons faire de toutes les manières.

\section{Clio : Dans votre enfance en Yougoslavie, vous semblez avoir été entourée de femmes fortes?}

MA : Oui, c'est pourquoi je ne me suis jamais sentie concernée par le féminisme, je ne savais rien de ce mouvement quand je demeurais en Yougoslavie parce qu'il y avait une égalité complète entre les hommes et les femmes dans l'armée des partisans. Mais vous savez, toute ma vie j'ai dû lutter avec cette présence de fortes femmes, ma mère, ma grand-mère et les autres, mais vraiment $j$ 'ai toujours pu faire ce que je voulais et je n'ai jamais senti d'opposition contre mes projets, tout était à ma disposition. J'ai été vraiment choquée quand j'ai découvert le féminisme aussi fort en Amérique, en Allemagne et dans le reste de l'Europe, c'était une idée complètement nouvelle pour moi. Et je n'y adhère toujours pas. Cependant, je dois avouer que dans ma vie privée, je sais aussi jouer avec la faiblesse; par exemple, si je vais à l'aéroport avec trois ou quatre valises, je joue à la femme fragile et regarde s'il n'y aurait pas un homme qui pourrait m'aider, mais s'il n'y a personne et bien tant pis, je le fais toute seule le plus souvent.

Clio : Dans Virgin-Warrior/Warrior-Virgin avec Ian Fabre, une performance donnée en 2004 au Palais de Tokyo, vous et votre partenaire Ian Fabre étiez couverts d'une armure médiévale ou nus. Pourriez-vous nous expliquer ce choix ?

MA : Ce n'était pas ma décision ; en fait, c'est Ian Fabre qui m’a invitée à participer à cette performance et l'armure est un élément qui revient souvent dans son œuvre, ce n'était pas mon choix. J'étais intéressée par une collaboration avec l'artiste de théâtre, mais je n'envisage plus une collaboration de longue durée avec un artiste. Ian Fabre a été influencé depuis longtemps par mon travail, en particulier dans ses premières performances, et il a toujours voulu que nous 
fassions quelque chose ensemble et moi-même j'étais intéressée de voir comment fondre nos deux univers dans le cadre d'une performance théâtrale et il a joué lui-même. C'était une expérience très intéressante. Il utilise très souvent les armures pour ses danseurs, pour ses installations comme celle du Louvre et de bien d'autres façons. C'était en fait son concept et pas mon choix.

Clio : Le culte du sacrifice et du pardon présent dans cette performance paraît très proche de deux archétypes : celui du saint et celui du guerrier ou du héros qui semble tous les deux vous fasciner, pourriez-vous nous expliquer les racines de cet intérêt ?

MA : En réalité, le plus intéressant pour moi dans une performance est le reflet des différentes projections sur la femme, c'était le thème de ma plus ancienne performance en 1975 appelée Written 0 où j'offrais mon corps comme objet pour six heures et où il y avait soixante douze objets sur une table que les personnes pouvaient utiliser sur moi, y compris un pistolet avec une balle. Et j'avais écrit un texte sur la table: "Je suis un objet, utilisez tous les objets que vous voulez, même la balle si vous voulez me tuer et j'en prends toute la responsabilité ». Elle a été donnée à Naples, dans le Sud de l'Italie, et ce qui est très intéressant, dans une galerie d'art normale. Le public qui venait était celui, tout à fait habituel, des galeries d'art. la performance a duré six heures et, pendant ce temps, tout ce qui a été fait sur moi était le reflet de la culture et des mentalités et de l'histoire de l'Italie, et les projections sur moi étaient celle de la mère, celle de la prostituée et celle de la vierge, la sainte Vierge : les trois projections typiques de cette culture. Et c'était très intéressant, très dur aussi.

Clio : Dans the Hero, une performance de $2001^{1}$, vous chevauchez un cheval blanc, en tenant un drapeau blanc et vêtue en noir, sans utiliser ici la nudité.

MA : Non, non, cette pièce était dédiée à mon père, c'est une autre histoire. Je l'ai réalisé quelques mois après sa mort et l'arrière-plan de

1 Voir Cahier d'images, figure 7. 
cette pièce est très simple. Comme vous le savez, mon père était aussi un héros national et sur toutes les photographies que je connais de la guerre, il était monté sur un cheval blanc. Mon père a eu le plus grand mal à accepter les changements politiques après la mort de Tito, avec la destruction complète du système communiste en Yougoslavie et la nouvelle guerre. Il a vraiment souffert et il en était venu à penser que son existence avait été inutile, qu'il n'avait contribué à rien et qu'on en était revenu au temps où les partisans étaient en prison pour communisme, comme dans les années 1930. Il était très déçu. Aussi, j'ai décidé de dédier cette pièce à mon père. Je suis assise sur le cheval blanc, personnification de son cheval blanc, et je porte un drapeau blanc. Un drapeau blanc, ici il y a une contradiction parce que la pièce s'appelle The Hero, et le drapeau blanc est la marque de la reddition. C'était une façon de dire à mon père de se rendre, de se rendre au changement. Et je ne voyais pas la nécessité de la nudité dans ce cas là. Parfois la nudité fait partie du concept, en particulier dans mes premières œuvres, des œuvres qui sont en rapport avec l'architecture et la simplicité du corps humain ou le son du corps frappant les murs. Mais ici le thème est celui de la reddition et du drapeau blanc qui flotte. La pièce est totalement statique, mis à part le vent dans le drapeau.

Clio : Le problème de la mémoire est central à l'existence du héros, mais aussi à celle du performer. Vous travaillez actuellement sur une autobiographie artistique?

MA : Je suis très intéressée par le problème de la mémoire, si vous pensez à la mémoire des performances ; par exemple pour celles réalisées dans les années 1970, tout ce qui reste le plus souvent, ce sont des images en noir et blanc ou des vidéos de mauvaise qualité car la technologie n'était pas encore développée ; c'est presque tout ce qui reste des années 1970, de tout ce qui s'est passé. On a bien sûr le souvenir des témoins qui ont assisté à ces performances et c'est à peu près tout; le reste n'est que spéculations ou mystifications sur ce qui aurait pu se passer dans les années 1970 et, de mon point de vue, les performances ne sont vécues que quand elles sont données. C’est pourquoi j'ai cette nouvelle attitude de redonner des performances 
historiques du passé et pas seulement les miennes, mais aussi de mes collègues comme Gina Payne, Bruce Newman, Victor Conci. C'est très important pour moi de trouver une nouvelle façon de redonner la performance qui prend ainsi une nouvelle vie ou qui peut être racontée par un témoin aux nouvelles générations sous la forme d'une narration. Pendant des siècles, la culture n'a pas été écrite, elle se transmettait de bouche à oreille, je pense que la mémoire et le témoignage sont aussi très importants, c'est ainsi que les exploits des héros ont été racontés de génération en génération.

\section{Clio : Particulièrement dans les Balkans?}

MA : Tout à fait, les chansons permettaient de se souvenir des héros, et c'est une mémoire collective, et la mémoire collective n'est jamais tout à fait exacte car elle ajoute toujours quelque chose de plus qui n'existe pas au début ; elle croît et elle tord la narration et peut aller jusqu'à en faire quelque chose de complètement différent. Mais de toute façon, n'importe quelle sorte de mémoire est meilleure que pas de mémoire du tout.

\section{Clio : Parfois, vous êtes un peu critique envers les artistes de la nouvelle génération qui donnent certaines de vos performances.}

MA : Non, vous savez, c'est juste une question de savoir comment cela se passe. Je suis la première artiste à avoir donné la permission de rejouer mes performances, mais beaucoup d'œuvres ont été jouées sans en respecter le fond, ;pas seulement les miennes, mais aussi celles de bien d'autres artistes. Par exemple, elles ont été utilisées pour des magasines de mode, sans tenir compte du contexte, à la télévision, pour la publicité de Loréal, au théâtre, pour de la danse. Vous voyez si vous utilisez n'importe quel morceau de Bach et que vous en faites de la techno Bach, vous devez préciser la source de ce matériau; si vous citez un livre, vous devez payer le copyright; la performance semble n'être le territoire de personne. Si je suis en colère, c'est vis-à-vis de ce manque de respect, mais si on mentionne la source de ce matériel ou la source d'inspiration et si vous 
demandez la permission à l'artiste vivant ou, si l'artiste est mort, à la fondation représentant l'artiste, c'est différent. Mais je voudrais donner mon avis aux jeunes artistes sur mon œuvre et leur dire : c'est ainsi qu'elle doit être jouée. Demandez la permission à l'artiste, regardez comment elle a été jouée et ensuite vous pourrez en donner votre propre vision, c'est la seule façon correcte de faire, malheureusement, personne ne le fait.

\section{Clio : C'est peut-être un problème inhérent à cette forme d'art ?}

MA : Oui, car la performance reste une forme d'art alternatif et la vidéo et la photographie sont restées longtemps alternatives jusqu'à ce qu'elles appartiennent aujourd'hui aux grands courants de l'art. La performance reste alternative, mais c'est ce qui fait sa vitalité, parce qu'elle est toujours nouvelle. Vous savez, je pense que ce moment de crise économique est très bon pour l'art, c'est la meilleure chose qui pouvait arriver à l'art car cela signifie que les vraies valeurs et les créateurs qui cherchent à créer resteront ; et la performance en particulier, parce qu'elle n'a rien de commercial.

\section{Clio : Existe-t-il un héroïsme de la vie quotidienne ?}

MA : Il y a dans la vie de chacun d'entre nous beaucoup d'actes héroïques. Il y a aussi beaucoup de petites morts autour de nous, durant notre vie, auxquelles on ne prête pas suffisamment attention. Il y a beaucoup d'héroïsme dans la vie quotidienne, en particulier dans la vie des femmes et surtout la somme de tous les sacrifices qu'elles font, sacrifices de leurs propres désirs pour leurs enfants ou leur mari ; et je ne suis pas d'accord avec cela. Il existe un concept tibétain très intéressant appelé "saint égoïsme »: cela signifie que vous devez avoir une certaine quantité d'égoïsme pour vous permettre de vous centrer sur vous-même, et c'est ainsi que vous pouvez aider les autres ; si vous n'en avez pas, et si vous passez votre temps à vous sacrifier pour les autres, vous vous perdez vous-même, vous devenez complètement inutile et vous ne pouvez plus aider les 
autres parce que vous vous perdez dans le sacrifice ; c'est pourquoi le sacrifice est très dangereux, cela semble contradictoire.

\section{Clio : Avez-vous encore le projet d'une Crucifixion avec une femme sur la croix?}

MA : Une des pièces des années 70 que je voulais rejouer était une pièce de Chris Burden qui se faisait crucifier derrière une Volkswagen et je lui ai demandé la permission mais il ne me l'a pas donnée, aussi j’ai respecté son choix. Pour moi, c'était intéressant.

Clio : Vous jouez souvent avec la peur, peur des insectes, peur des serpents, mais aussi avec le sadisme ou la peur éprouvés par les spectateurs. La peur est-elle un élément essentiel du processus de création?

MA : C'est important pour l'être humain d'être libéré de la peur. On a deux grandes peurs basiques dans la vie, celle de souffrir et celle de mourir. Pour ma part, je ne suis pas concernée par ces peurs dans ma vie privée, mais je les joue en face du public et utilisant l'énergie du public. Je me confronte aves ces peurs, mais le public peut aussi se voir en moi comme dans un miroir et si je peux le dépasser et m'y confronter, les spectateurs peuvent aussi le faire dans leur propre vie ; c'est une image inversée qui me permet de communiquer avec le public et je peux seulement le faire si je le joue sous la forme d'une performance. Dans ma vie privée, je ne cherche pas à souffrir mais, pendant la performance, je le joue pour m'y confronter, pour montrer qu'elles existent, qu'on peut s'y confronter, les adoucir et les surpasser. Si je peux souffrir pour me libérer de la souffrance, alors j'accepte la souffrance et c'est très important. Mais c'est un outil pour créer une œuvre d'art.

Vous savez, cela n'a rien de nouveau car dans les cultures anciennes, toutes sortes de cérémonials, par exemple dans les approches chamanistes du corps, sont basés sur la souffrance. La médecine chamaniste va même jusqu'à faire face à la mort clinique pour explorer ce passage entre vie et mort. C'est très important car vous 
comprenez les forces de l'esprit et la mortalité du corps physique. Ces choses sont essentielles, nous les oublions parce que dans la vie quotidienne on est concerné par les choses terrestres et l'on oublie les grandes choses ; nous oublions que nous sommes mortels et pas immortels. Mais adopter un autre point de vue est très important pour moi et c'est le moment, ici et maintenant, le présent qui compte, car on ne peut pas regarder vers le passé ; il a déjà disparu et l'on ne sait rien sur un futur incertain. La seule chose dont on peut être certain, c'est le présent et la performance est basée sur le moment. C'est pourquoi c'est pour moi une forme d'art très importante, parce que c'est ici et maintenant et une seconde plus tard, la pièce peut s'effondrer et nous serons tous morts. Seul le présent est certain, le moment présent.

\section{Clio : Pourquoi votre performance Cleaning the floor?}

MA: Vous savez, j'aime beaucoup rejouer des moments très dramatiques de ma vie. J'avais des amis et ils sont morts dans un accident d'avion et quand je suis arrivée dans leur maison, tout était en place comme avant: les pommes sur la table, la photographie des enfants dans l'autre pièce. C'était si triste, si incroyablement triste la présence de tous ces objets sans aucun être vivant, un accident d'avion et tout disparaît ; et la seule chose que j'ai trouvé à faire c'est de prendre une bassine et de laver le sol dans toute la maison et c'était un souvenir très fort. Laver le sol est une sorte d'acte métaphorique, un acte pour comprendre vraiment la versatilité de la vie, que la mort peut arriver à n'importe quel moment. Cela n'a rien à voir avec la femme victime, la ménagère, cela ne m’intéresse pas. J'ai pris un baquet d'eau et des gants rouges pour donner une certaine modernité à l'image.

Vous savez, c'est une chose très présente dans mon œuvre, j'ai lavé mon image dans un miroir, j’ai lavé mon propre squelette, laver est un grand sujet pour moi. Laver à l'intérieur, laver l'âme c'est très important, laver le miroir ou votre propre squelette, pour se confronter avec sa mort. Je suis allée en Allemagne et j'ai fait faire un squelette à ma taille, mon propre squelette pour voir à quoi je 
ressemblerai. Il y a une vieille tradition au Tibet où les moines dorment dans un cimetière avec les corps à des stades différents de décomposition : ceux qui sont morts la veille, ceux qui sont morts depuis dix jours, ceux qui sont dévorés par les vers, ceux qui sont tout décomposés mangés par les oiseaux et les animaux, ceux dont il ne reste plus que les ossements et ceux qui sont tombés en poussière. C'est important de voir ce qui va réellement vous arriver, c'est une terrible confrontation, une confrontation directe et c'est aussi ce que j'essaie de faire dans mon œuvre, car on ne veut pas savoir, on veut toujours ne rien voir. Ne pas voir la réalité.

$\mathrm{Ma}$ fonction en tant qu'artiste est de témoigner pour le public, de lui donner conscience, mais aussi de faire vivre l'esprit; oui nous sommes mortels, mais il y aussi l'énergie, l'esprit, montrer l'esprit. Car c'est si facile d'oublier le spirituel, très facile, on le fait tout le temps.

\section{Clio : L'héroïne a-t-elle une place dans la vie quotidienne ?}

MA : C'est très intéressant de savoir que dans le passé, l'héroïne est toujours une figure tragique, mais je voudrais vous raconter une autre histoire merveilleuse que j'aime tant, un peu différente, celle de sainte Thérèse d'Avila, la sainte espagnole. Dans son journal, elle raconte les nombreuses fois où elle est entrée en lévitation, et une fois elle raconte qu'elle avait lévité toute la journée dans l'église et quand elle est revenue chez elle, elle avait faim et a voulu se faire une soupe mais elle ne pouvait contrôler la force divine ; elle a lévité et a regardé la soupe brûler sans pouvoir rien faire ; c'est une de mes histoires favorites. Finalement, la lévitation a quelques légers désavantages. Je voudrais faire une œuvre en hommage à sainte Thérèse, appelée Soupe et Lévitation ou Cuisine et Complainte.

\section{Clio : Quels sont vos projets pour la fondation Marina Abramovic à Hudson?}

MA : Elle s'appellera l'institut Marina Abramovic pour la préservation de l'art de la performance, je veux seulement laisser mes idées. Le but est de créer un institut pour la performance sous toutes ses formes, 
l'opéra, la danse, la vidéo, les films et les performances. Ce qui fera la différence entre cet institut de la performance et les autres dans le monde, c'est une grande différence : la longueur des performances; elles devront durer au moins six heures et plus. Mais pas moins de six heures. La vie va de plus en plus vite, moi, je veux rendre l'art de plus en plus long et surtout les performances. Je voudrais qu'on installe des sièges spécifiques avec des dossiers inclinables, toutes sortes de commodités. Il y aura une école pour les jeunes artistes intéressés par la performance, une commande pour les artistes qui souhaitent créer ce type de performances, des symposiums pour les nouveaux développements en science et technologie. Ce serait la différence avec tous les autres instituts et c'est mon rêve. La seule chose que nous avons de moins en moins dans le monde moderne, c'est l'espace et le temps, et surtout le temps.

\section{Clio : Avez-vous des héros, hommes ou femmes ?}

MA : Si j’ai des héros ? Oh, c'est étrange. Vous savez quand vous êtes enfant, vous avez ces idées de héros, des jouets, je n'ai jamais eu de jouets, je ne me souviens d'aucun jouet. Je ne peux pas dire que j'ai jamais été influencée par un artiste, parce que je ne souhaitais pas être influencée, ni par aucun héros. La seule chose qui m'ait vraiment intéressée, c'est la cosmologie, les sciences, l'anthropologie, la connaissance des anciennes civilisations, c'était mes influences, mes sortes de rêves, mais ce n'était pas les vraies personnes. Ce que je voulais, c'était aller aux sources, pas une information de seconde main. Bien sûr, j'aime sainte Thérèse, c'est en quelque sorte mon héroïne, mais je ne vois pas quelqu'un de spécifique qui m'ait influencée, même pas Jeanne d'Arc.

Ce qui m'a beaucoup impressionnée depuis longtemps et que je voudrais vous décrire, c'est l'image de Kali, la déesse indienne. Une très ancienne image la représente dansant avec jambes et bras multiples : d'une de ses mains, elle coupe sa tête avec une épée et elle la tient, mais de son cou s'écoulent trois fontaines de sang, l'une coule vers sa bouche et la nourrit et les deux autres coulent sur deux dévots à ses pieds, un homme et une femme qui ouvrent la bouche. Elle 
donne ainsi la vie à trois choses, sa tête coupée et les deux dévots. Elle danse sur les corps de deux autres dieux, homme et femme qui font l'amour et cet acte sexuel donne à son corps l'énergie pour vivre et ne jamais mourir. Le sang fait un circuit complet dans son corps ; c'est une des images les plus étonnantes pour moi, qui dégage le plus d'énergie. Une image sur laquelle vous pouvez méditer de manières si différentes, un cycle complet qui ne s'arrête jamais. On pense que la mort est une fin, mais les soufis disent que la vie est un rêve et que la mort, c'est se réveiller. C'est l'une des images de femmes les plus fortes, de déesse femme, de l'idée féminine du cycle sans fin. C'est sans doute l'une des influences les plus fortes pour moi.

Clio : Peut-être est-ce la raison pour laquelle vous n'avez pas de héros, car le héros est celui par qui le changement arrive, qui produit l'avènement du futur?

MA : Et ensuite, il meurt. Pour moi, la connaissance orientale est fondamentale, je cherche à la transformer dans ma propre conception. Le concept de temps, vie et mort, est quelque chose que j'ai du mal à comprendre. Tout est interdépendant dans la vie. Il n'y pas de début clair et de fin définie. D’une certaine manière, le catholicisme et l'orthodoxie sont des religions qui commettent de grandes erreurs, ce sont des institutions, des pouvoirs politiques, elles ne sont plus dans la vérité. Mais, vous savez, mon grand-père était un saint.

\section{Clio : Vous avez des héros et des saints dans votre famille?}

MA : Oui, mes deux parents étaient des héros nationaux, des partisans,communistes complètement antireligieux, ils étaient athées tous les deux. Mon grand-père était le patriarche de l'Eglise orthodoxe de Serbie ; n 1937, le roi de Serbie a souhaité l'unification du catholicisme et de l'Eglise orthodoxe de Serbie et il a refusé. Il a été invité à un repas par le roi avec ses deux frères, et durant ce repas, sur l'ordre du roi, le médecin privé du roi leur a donné dans la nourriture du diamant réduit en poudre ; les trois sont morts les uns 
après les autres de terribles hémorragies à l'estomac. Et après, il a été proclamé saint par l'Église orthodoxe de Serbie et il est encore vénéré en Serbie. C'est le patriarche Barnaba.

C'est vrai que je suis issue de bien des contradictions. Quand j'étais petite, j’ai été élevée par ma grand-mère jusqu'à l'âge de six ans, mon père et ma mère étant occupés par leur carrière politique ; et comme ma grand-mère était tout le temps à l'église, je vivais au milieu des prêtres. Et ensuite, avec mes parents, on ne parlait plus que de Lénine et de Staline.

Clio : Votre histoire personnelle semble donc très liée à l'histoire de votre pays?

MA: C'est vrai, mais je ne me suis jamais sentie typiquement yougoslave, je suis plutôt attachée à la planète en général. J'ai habité en Yougoslavie sans être une artiste typiquement yougoslave, j’ai vécu en Hollande avec un passeport hollandais, j'ai travaillé en Allemagne, j’ai enseigné ici à Paris aux Beaux Arts pendant 4 ans, et maintenant j'habite à New York (Hudson) depuis 7 ans ; mais ce n'est pas très important, car ce que j'aime, c'est la vie que j'appelle « être entre »; quand vous êtes au milieu de tout, le meilleur endroit, c'est l'avion.

Clio : Mais dans le texte de The Onion, vous semblez épuisée par cette vie trépidante et vous psalmodiez une véritable lamentation sur la vie moderne?

MA : Ce n'est qu'un moment de vie, une semaine et demie où j'ai pris quinze vols internationaux ; quand je suis arrivée à New York, je suis allée au cinéma et machinalement j’ai essayé d'attacher une ceinture de sécurité, j'étais complètement perdue, je ne savais plus où j'étais. Il $\mathrm{y}$ a des moments de tristesse, des moments de joie, et cette exposition est la somme de tous ces moments et les bénéfices de cette exposition iront à ma fondation. 


\section{Bibliographie sommaire}

FisHER Jennifer, 1997, "Interperformance: The Live Tableaux of Suzanne Laci, Janine Antoni and Marina Abramović », Art Journal 56, p. 28-33.

GreEN Charles, été 2000, «Doppelgangers and the Third Force: The Artistic Collaborations of Gilbert \&George and Marina Abramović/Ulay », Art Journal $59 / 2$, p. 36-45.

KAPLAN Janet, 1999, «Deeper and Deeper: Interview with Marina Abramović », Art Journal, 58:2, p. 6-19.

Kosmidou Zoe, 2001, «A Conversation with Marina Abramović », Sculpture, p. 2731.

Novakov Anna, 2003-2004, "Point of Access: Marina Abramović's 1975 Performance Role Exchange », Woman's Art Journal, p. 31-35. 